\title{
Association Between Dexamethasone and Delirium in Critically ill Patients: A Retrospective Cohort Study of a Large Clinical Database
}

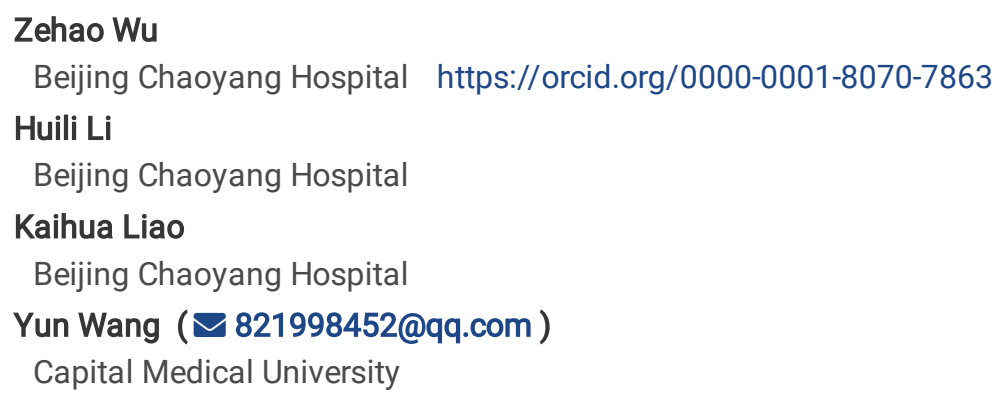

Research

Keywords: Dexamethasone, Intensive care unit (ICU), Delirium, Mortality, Propensity score matching (PSM)

Posted Date: October 13th, 2020

DOI: https://doi.org/10.21203/rs.3.rs-89771/v1

License: () (1) This work is licensed under a Creative Commons Attribution 4.0 International License. Read Full License

Version of Record: A version of this preprint was published at Journal of Surgical Research on July 1st, 2021. See the published version at https://doi.org/10.1016/j.jss.2021.01.027. 


\section{Abstract}

Background

Delirium is a common complication in ICU patients, and it can significantly increase the length of hospital stay and cost. Dexamethasone is widely used in various inflammatory diseases and is a glucocorticoid commonly used in critically ill patients. There are no studies on the effect of dexamethasone on the development of delirium in critically ill patients, therefore, this study aimed to confirm the effect of dexamethasone use and the dose on the incidence of delirium and patient prognosis in critically ill patients through a large cohort study.

Methods

A retrospective cohort study was conducted using data extracted from the MIMIC III database, and the primary outcome was the development of delirium, using multivariate logistic regression analysis to reveal the relationship between dexamethasone and delirium. Secondary endpoints were in-hospital mortality, total length of stay and length of ICU stay, and the relationship between dexamethasone and prognosis was assessed with Cox proportional hazards models. The Lowess smoothing technique was used to investigate the dose correlation between dexamethasone and outcomes, subgroup analysis was used to account for heterogeneity, and different correction models and propensity matching analysis were used to eliminate potential confounders.

Results

Finally, 38,509 patients were included, and 2,204 (5.7\%) used dexamethasone. A significantly higher incidence of delirium (5.0\% vs. 3.4\%, $P<0.001)$, increased in-hospital mortality $(15.0 \%$ vs. $11.3 \%, P<0.001)$, and longer length of stay and ICU stay were observed in patients taking dexamethasone compared with those not taking dexamethasone. Multivariate logistic and Cox regression analyses confirmed that dexamethasone was significantly associated with delirium (adjusted $O R=1.45,95 \% \mathrm{Cl}=1.08-1.95, \mathrm{P}=0.014$ ) and in-hospital mortality (adjusted HR $=1.19,95 \% \mathrm{Cl}=1.02-1.40, \mathrm{P}=0.032$ ). The risk of delirium and in-hospital death was lower with dexamethasone less than $10 \mathrm{mg}$, and subjects with 10-14 mg had the shortest length of hospital stay.

\section{Conclusions}

This study demonstrated that the use of dexamethasone in critically ill patients exacerbated the occurrence of delirium, while increasing the risk of in-hospital death and length of stay, and the use of low-dose dexamethasone had a lower risk of delirium and death, which appeared to be safer.

\section{Key Points}

- Question: Previous controversies have been inconclusive about the effect of dexamethasone on postoperative delirium, so in critically ill patients of ICU, does the use and dose of dexamethasone have an effect on the occurrence of delirium?

- Findings: The use of dexamethasone in critically ill patients exacerbates the development of delirium, while increasing the risk of inhospital mortality and length of stay, and low-dose dexamethasone had a lower risk of delirium and death.

- Meaning: To reduce the risk of delirium, it may be of interest to reduce the use of nonessential dexamethasone and control the intake level of dexamethasone in ICU patients.

\section{Introduction}

Delirium is a neuropsychiatric disorder that also refers to a group of syndromes, known as acute brain syndrome. It is characterized by sudden onset of changes and fluctuations in the level of consciousness, inattention, and confusion [1]. Almost all previous studies have targeted postoperative delirium and investigated the effect of perioperative interventions such as surgery and anesthesia on the occurrence of postoperative delirium [2-5]. The pathogenesis of postoperative delirium is complex, multifactorial, and not yet fully understood, but multiple risk factors have been identified, including age, previous psychiatric illness, cerebrovascular disease, previous cognitive impairment, and type of surgery [6, 7]. However, a large proportion of critically ill patients who do not undergo surgery still develop delirium, which is not uncommon in various intensive care units (ICUs) [8-10], with mild disturbance of consciousness, severe memory loss and even death, which leads to prolonged ICU stay and hospital length of stay (LOS), increasing medical costs and unnecessary pain $[11,12]$. Therefore, it is particularly important to investigate the causes of delirium in critically ill patients in the ICU and intervene. 
There is increasing evidence that the response of the brain to peripheral inflammation may play a role in the development of postoperative cognitive dysfunction (POCD) and postoperative delirium (POD) [13, 14]. Dexamethasone (DEX) is a long-acting glucocorticoid widely used in various inflammatory diseases [15]. If the inflammatory reaction plays a role in the development of POD and POCD, then inhibiting the inflammatory response by DEX may prevent the development of POD and POCD [14, 16]. However, other reports have demonstrated that high-dose oral glucocorticoid therapy increases the risk of psychiatric events, such as mood disorders, anxiety, aggressive behavior, insomnia, irritability, depersonalization, panic disorder, suicidal thoughts, dementia, and delirium [17, 18]. A number of previous studies have investigated the effect of DEX on POD and POCD, but their conclusions are inconsistent and inconclusive [17, 19-21]. There are no studies on the effect of DEX on the development of delirium in critically ill patients, therefore, we assessed the effect of DEX use and the dose on the incidence of delirium and patient prognosis in critically ill patients through a large retrospective cohort study.

\section{Methods}

\section{Study design and data source}

This study used a retrospective observational cohort study design. Study data was extracted from Medical Information Mart for Intensive Care III (MIMIC III) database, which is a large and freely available database that included 46,476 patients in the intensive care unit at Beth Israel Deaconess Medical Center, Boston, MA, USA, from 2001 to 2012 [22]. Public de-identified databases were used for this study, so informed consent and institutional review board (IRB) approval was waived. We were granted access to the database after completing a web-based training course based on the Cooperative Institutional Training Initiative (CITI program) entitled 'Data or Specimens Only Research' (Record ID: 36171671). This access was approved by the Institutional Review Boards of Beth Israel Deaconess Medical Center and MIT affiliates. The clinical data required for this study, including patient demographics and laboratory test results, were extracted by the author Wu.

\section{Population selection criteria}

Patients included in the cohort should meet the following inclusion criteria: (1) age $\geq 18$ years; (2) length of hospital or ICU stay $>1$ day. If there were more than one admission record in the database, only patients who were hospitalized for the first time were selected. Excluded were any of the following: (1) DEX use before or after admission; (2) DEX use without clear dosage specification; and (3) patients who had already experienced or had experienced delirium before admission. In addition, to avoid potential input errors in the raw data, we excluded patients with a hospital stay shorter than ICU LOS. The detailed flow chart is shown in Figure 1.

\section{Data extraction}

Data were extracted from MIMIC III using Structured Query Language (SQL) with PostgreSQL (version 9.6) [23]. The extracted data included age, gender, ethnicity, height, weight, type of ICU on admission, use and dosage of DEX, and occurrence of delirium. Body mass index (BMI) was calculated as weight $(\mathrm{kg})$ divided by height $(\mathrm{m})$ squared. Complications included congestive heart failure (CHF), coronary artery disease (CAD), hypertension, chronic obstructive pulmonary disease (COPD), acute respiratory failure (ARF), diabetes, liver disease, acute kidney injury (AKI), cerebrovascular disease, sepsis. Laboratory parameters include red blood cell (RBC), hemoglobin, white blood cell (WBC) and platelet counts, sodium, potassium, calcium, serum bicarbonate, serum creatinine, blood urea nitrogen (BUN), glucose, and blood lactate values. Severity of illness was measured using the Sequential Organ Failure Assessment (SOFA) score [24] and the Acute Physiology Score III (APS III) systems [25]. Baseline characteristics were extracted within $24 \mathrm{~h}$ of ICU admission.

\section{Propensity score matching (PSM) and stratification method}

Since many confounding factors in the baseline indicators had an effect on the initial cohort, which reduced the comparability of outcome measures between the two cohorts, we tried to calculate the propensity score of each patient according to the following covariates by using the multivariate logistic regression model, and grouped them 1:1 according to the score to eliminate the effect of confounding factors on the two cohorts: age, gender, ethnicity, BMI, ICU type, SOFA score, APS III, CHF, CAD, hypertension, COPD, ARF, diabetes, liver disease, cerebrovascular disease, sepsis, RBC, WBC, hemoglobin and platelet count, sodium, potassium and calcium level, creatinine, BUN, blood glucose and lactate value.

In order to further investigate the effect of DEX dose on the occurrence of delirium, logistic regression analysis and comparison of Lowess curves were performed by dividing the total DEX dose in patients using DEX into five levels by quintiles: level $1(0-4 \mathrm{mg})$, level 2 
(4 - $10 \mathrm{mg})$, level $3(10-14 \mathrm{mg})$, level $4(14-23 \mathrm{mg})$, and level $5(23-100 \mathrm{mg})$. Because the two cohorts remained heterogeneous for ICU type after PSM analysis, we further performed a subgroup analysis according to the type of ICU admitted.

\section{Outcomes}

The primary outcome is whether patients admitted to the ICU develop delirium, which was defined as critically ill patients developing or being diagnosed with delirium in the ICU. Secondary endpoints included in-hospital mortality, hospital LOS, ICU LOS, defined as death during hospitalization, total LOS, and total ICU LOS, respectively. Due to patients may be admitted to the ICU more than once during one hospitalization, only various outcome measures for patients' first ICU admission were included.

\section{Statistical analysis}

Tabulated continuous variables are expressed as mean and standard deviation (SD) or median and interquartile range (IQR). The t-test was used for variables that obeyed normal distribution, and the Wilcoxon rank-sum test or Kruskal-Wallis test was used for skewed distribution. Categorical variables were presented as $n(\%)$ and compared using the $\chi^{2}$ (or Fisher's exact) test. Baseline differences between the two cohorts were balanced using PSM.

Logistic regression models were used to predict the association between DEX and delirium, and Cox regression models were used to assess the association between DEX and in-hospital mortality. We used three different models to adjust for potential confounders affecting the primary and secondary outcomes: (1) Model 1, including age, sex, ethnicity, BMI, and type of ICU on admission; and (2) Model 2, which added comorbidities and laboratory tests to Model 1, including CHF, CAD, hypertension, COPD, ARF, diabetes, liver disease, cerebrovascular disease, sepsis, RBC, WBC, hemoglobin, platelets, sodium, potassium, calcium, creatinine, BUN, blood glucose, lactate, SOFA score, and APS III; (3) Model 3, a propensity score as mentioned above. To compare the effect of DEX on outcome measures in different ICUs before and after PSM by subgroup analyzing ICU types with greater heterogeneity. Lowess Smoothing technique were further used to investigate the crude relationship between DEX dose and delirium and in-hospital mortality. The final model was developed using a stepwise backward elimination method with a significance level of 0.05 . All logistic regression models were assessed for goodness-of-fit.

A two-sided $p$ values $<0.05$ of each test was considered statistically significant. All statistical analyses were conducted using software Stata 15.1 (StataCorp, College Station, TX).

\section{Results}

\section{Characteristics of patients}

We included 38,509 patients in the study, of whom 2,204 (5.7\%) used DEX during hospitalization and the remaining 36,305 (94.3\%) did not. Table 1 enumerates the baseline characteristics of the original two-group cohort and the post-PSM two-group cohort. The baseline levels of age, gender, race, BMI, type of ICU admission, SOFA score, APS III, CHF, CAD, hypertension, COPD, ARF, diabetes, liver disease, cerebrovascular disease, sepsis, RBC, WBC, hemoglobin, platelet, sodium, potassium, calcium, creatinine, BUN, glucose and lactate were statistically significantly different between the two groups before PSM $(p<0.05)$. Using PSM (1:1 matching ratio), 2,204 patients who used DEX were matched with 2,204 patients who had not used DEX in the hospital. After matching, the imbalance was significantly reduced in both cohorts, and all baseline variables were comparable between the two groups except for ICU type.

\section{Primary outcome}

A crude comparison of the primary outcome was first made, and as shown in Table 2, before matching, the incidence of delirium was $5.0 \%$ vs. $3.4 \%$ in the DEX vs. non-DEX group ( $<0.001$ ); after matching, the occurrence of delirium remained significantly higher in the DEX group than in the control group $(5.0 \%$ vs. $3.5 \%, p=0.014)$. Further, three multivariate logistic regression models were established to test the difference in the occurrence of delirium between the two groups. The results showed that the use of DEX in critically ill patients was a risk factor for the occurrence of delirium, with a crude odds ratio $(\mathrm{OR})$ of 1.51 [95\% confidence interval $(\mathrm{Cl})=1.23-1.84]$. After adjustment for three different models, the association was still significant and consistent $(\mathrm{OR}=1.45,95 \% \mathrm{Cl}=1.08-1.95$, adjusted for model 3; the results of the other two models were presented in Table 3). In addition, in order to investigate the effect of different doses of DEX on the occurrence of delirium, we roughly equally divided the patients using DEX into five groups using quintiles as the cut-off value, and established a Lowess curve based on a logistic regression model, and preliminarily found that the incidence of delirium also showed 
a positive trend increase with increasing doses of DEX, and low-dose DEX $(<10 \mathrm{mg}$ ) could significantly reduce the risk of delirium compared with medium and high doses $(10-100 \mathrm{mg})$. (Figure 2A)

\section{Secondary outcomes}

Crude differences in hospital mortality between the two cohorts in secondary outcomes are shown in Table 2, with patients in the DEX group having significantly higher hospital mortality than patients in the non-DEX group before matching $(15.0 \%$ vs. $11.3 \%, p<0.001)$; after matching, there was no statistically significant difference between the two cohorts $(15.0 \%$ vs. $13.6 \%, p=0.182)$. Further, three Cox proportional hazards models were established to test the difference in hospital mortality. The results showed that the use of DEX in critically ill patients was a risk factor for hospital death, with a crude hazard ratio (HR) of 1.45 [95\% $\mathrm{Cl}=1.29-1.62]$. After adjustment for three different models, the association was still significant and consistent $(\mathrm{HR}=1.19,95 \% \mathrm{Cl}=1.02-1.40$, adjusted for model 3 ; the results of the other two models were presented in Table 3). In addition, by Lowess smoothing technique, we initially found that the use of too little or too much DEX significantly increased hospital mortality. (Figure 2B)

It is easy to see from Table 2 that the hospital and ICU LOS were significantly longer in patients who used DEX than in those who did not, both before and after matching. Figure 2 roughly confirms by dose correlation that patients using $10-14 \mathrm{mg} D E X$ achieved the shortest hospital and ICU LOS. (Figure 2C, D)

\section{Subgroup analysis}

As shown in Table 4, the estimates of the association between DEX use and the development of delirium were broadly consistent among patients with different types of ICU stay after stratification by ICU type of admission. However, estimates of the association between DEX and in-hospital mortality varied after subgroup analysis according to ICU type, with a much higher risk of in-hospital mortality (adjusted for model 3) observed with DEX use among patients in the cardiac surgery recovery unit (CSRU) $(\mathrm{HR}=2.70,95 \% \mathrm{Cl}=1.06-6.84)$ and medical intensive care unit (MICU) $(\mathrm{HR}=1.78,95 \% \mathrm{Cl}=1.42-2.22)$, whereas a significant negative association was observed among patients hospitalized in the surgical intensive care unit $(\mathrm{SICU})(\mathrm{HR}=0.38,95 \% \mathrm{Cl}=0.25-0.55)$.

\section{Discussion}

This observational study is the first to investigate the impact of DEX use and dose in critically ill patients on delirium development and patient prognosis. This is also one of the largest studies conducted in terms of the number of patients included. This retrospective cohort study showed that the use of DEX in critically ill patients could promote the occurrence of delirium, and increase the in-hospital mortality, total hospital and ICU LOS; the use of DEX less than $10 \mathrm{mg}$ had the lowest risk of delirium and death; the use of 10-14 mg could obtain the greatest benefit in the length of hospital stay. From the results, DEX has the risk of increasing the occurrence of delirium in ICU patients, and the risks and benefits need to be reasonably considered when used. If it is necessary to use, low-dose DEX may be safer when the treatment needs are met.

In the hospital setting, the prevalence of delirium in general medical patients is estimated to be between $10 \%$ and $30 \%$, while it is even higher estimated to be $80 \%$ in patients in the ICU $[26,27]$. Fortunately, up to $40 \%$ of delirium is preventable [28]. Delirium may be caused by different pathophysiological mechanisms, including disturbances in several neurotransmitter systems of the brain, which are not fully understood. Previous studies by Mardani et al [19] have shown that DEX is a protective factor for POD in cardiac surgery. In cardiac surgery, excessive inflammatory cytokines flowing into the brain due to extracorporeal circulation and other factors can cause neurological and neuroendocrine system dysfunction, resulting in a high incidence of POD in cardiac surgery. DEX and its regulatory effect on inflammatory mediators (proenzyme activator, IL-6, IL-8 and TNF-a) disrupt the above functions, thereby reducing the incidence of POD in cardiac surgery $[19,29,30]$.

However, there are a large number of studies demonstrating that the occurrence of POD in patients using DEX is not significantly different from that in patients not using it $[14,20,31,32]$. The occurrence of POD is the result of the interaction of multiple factors, and a single intervention cannot completely affect it. Although there is evidence that inflammation is an important pathogenesis of POD, other factors that have not been clarified may play a greater role in the occurrence and development of POD [14, 33], such as surgical factors, anesthetic drugs, especially benzodiazepines, which have been shown to seriously affect and exacerbate the occurrence of POD [31, 34, 35], and these factors are not present in most non-surgical critically ill ICU patients. It also explains why treatment with other antiinflammatory drugs such as statins [36] and haloperidol $[37,38]$ does not appear to reduce the incidence of POD. Therefore, the effect of DEX on delirium in non-surgical critically ill patients was previously unknown. 
Corticosteroids used to treat many diseases cause some systemic adverse effects, some of which are psychiatric side effects [39, 40]. An earlier study reported that more than half of 55 patients $(n=30,54.5 \%)$ who had used different types of steroids were diagnosed with mental disorders, and the mean duration of delirium was 7 days [18]. Glucocorticoids may play an important role in the pathogenesis of delirium, especially in elderly patients [41]. Abnormal "shutdown" of the hypothalamic-pituitary-adrenal (HPA) axis appears to be a key abnormal link in the development of delirium. When glucocorticoid levels increase, they act on a more restricted portion of the HPA axis to prevent stress-induced further hormone secretion [42]. In addition, an intact hippocampal formation is essential for the closure of the normal HPA axis $[43,44]$. In this brain region, there is a close interaction between neurotransmitters (especially acetylcholine, serotonin, and norepinephrine) and glucocorticoid receptors, which may be associated with delirium in elderly patients with stroke and neurodegenerative encephalopathy [45-47]. DEX has a biphasic effect on several physiological functions of neurons, which can be extended to effects on memory, particularly on hippocampal-related visuospatial memory. Even moderate doses of DEX may impair "hippocampal-like" declarative memory performance [48, 49]. Lupien et al showed that elderly subjects with elevated circulating cortisol levels experienced a decline in hippocampus-dependent memory with a 14\% reduction in hippocampal volume over 5 to 6 years of follow-up [50]. DEX may aggravate psychiatric conditions in certain pathological disorders (e.g., Cushing's syndrome, Alzheimer's disease), which can lead to HPA axis dysregulation [21,51,52]. In addition, the study found that $5 \%$ of patients developed glucocorticoidinduced psychosis or mania, and the mean albumin level in these patients was significantly lower than that in patients who did not develop mental disorders, while critically ill patients admitted to the ICU had prevalent albumin levels [53, 54]. So, in order to reduce the symptoms of delirium, it may be of interest to control the intake level of DEX.

Studies have shown that the most important risk factor for psychiatric symptoms after DEX use is the dose used. Early studies from the Boston Collaborative Drug Monitoring Program confirmed significant dose-response associations for glucocorticoids [55]. Chan et al showed that $8 \%$ of patients taking $90 \mathrm{mg}$ prednisone per day had psychosis, compared with only $3 \%$ of patients taking $30 \mathrm{mg}$ per day [56]. The effect of DEX on delirium is also dose-dependent. DEX is a long-acting glucocorticoid with an extended half-life of 36 to 54 hours that will result in more drug accumulation over time compared to methylprednisolone with a half-life of 18 to 36 hours [57]. Therefore, repeated high-dose DEX use in a short period of time may result in more drug accumulation than expected, which also explains the observation that the higher the dose of DEX is used in critically ill patients, the higher the risk of delirium or in-hospital death. Specifically, there was an "inverted U-shaped" dose response to the effect of DEX on psychiatric symptoms, meaning that very low or high levels of serum cortisol (modulated by DEX levels) can cause the brain to exhibit symptoms such as cognitive impairment and delirium $[21,51,58]$, which in turn exacerbates the risk of death in patients, consistent with our observed dose effect on mortality outcomes. In the study by Genis et al., patients did not experience psychiatric symptoms when taking oral DEX $20 \mathrm{mg}$, while when the dose was increased to $40 \mathrm{mg}$, patients developed psychiatric symptoms within the first week [54].Similarly, it has been shown that side effects caused by psychosis are associated with prednisone $>80 \mathrm{mg}$ /day (equivalent to $13 \mathrm{mg} \mathrm{DEX)} \mathrm{and} \mathrm{declarative} \mathrm{memory} \mathrm{loss}>40 \mathrm{mg}$ (equivalent to 6 $\operatorname{mg} D E X)[55,59,60]$. A large multicenter trial showed that intraoperative high-dose DEX had a significant effect on the risk of delirium development [61, 62]. In addition, low-dose DEX $4 \mathrm{mg} /$ day improved depressive symptoms in patients with major depressive disorder compared to placebo $[63,64]$. Thus, the chance of developing delirium may also increase with increasing doses of DEX in critically ill patients regardless of their psychological history.

Our study showed that critically ill patients using DEX had prolonged hospital and ICU stays (especially with high-dose use), and this result may be caused by the high incidence of delirium in this part of the patients, as Hopkins et al [65] showed that patients who developed cognitive impairment and delirium had a 2.7-fold higher risk of total ICU stays of more than 27.4 days than those who did not. In addition, our subgroup analysis showed that ICU admission type was not a source of heterogeneity, and the use of DEX in various ICUs increased the risk of delirium development. In terms of death, only the use of DEX in SICU reduces the risk of in-hospital death. In combination with the lower OR of delirium in patients using DEX in SICU compared with other ICUs, we infer that the benefit may be higher in patients using DEX in SICU than in other ICUs, which may be related to that most patients in SICU are surgical patients. Some previous studies have shown that DEX has a protective effect in surgical patients $[19,21,66]$.

This study has several limitations. First, patients in the MIMIC III database may not have a perfect baseline level affecting delirium before admission, including preoperative cognitive status, educational level, sensory disturbance and psychiatric history, etc., and these original values may not be completely recorded in this database. Although these factors may be relatively rare in critically ill patients, we cannot exclude that there may be some imbalance between the two cohorts, for which we performed a series of model efforts to correct for the effect of confounding factors reducing the imbalance. Second, in addition to clinical parameters and comorbidities, patients were only followed up for a short period of up to 90 days after discharge, while the 90-day follow-up period could not fully determine the outcome of delirium patients, so our study did not involve the long-term prognosis of delirium in critically ill patients using DEX. So, prospective studies are needed to further confirm our results. Third, our study was a retrospective design. Despite careful PSM and further subgroup 
analysis for the presence of heterogeneity, residual confounding cannot be completely ruled out. Therefore, the risk of confounding factors is considered in the interpretation of results. Finally, an observational study based on single-center electronic historical records, and the results need to be verified by a multicenter trial.

\section{Conclusions}

In summary, in this large retrospective analysis, we confirmed that the use of DEX in critically ill patients may exacerbate the development of delirium while increasing the risk of death and length of hospital stay of patients during hospitalization. In addition, compared with high-dose, low-dose DEX has lower risk of delirium and death and safer. These results also need to be validated by multicenter randomized controlled trials in the future.

\section{Declarations}

\section{Ethical approval and consent to participate}

Study data was extracted from Medical Information Mart for Intensive Care III (MIMIC III) database, which is a large and freely available database that included 46,476 patients in the intensive care unit at Beth Israel Deaconess Medical Center, Boston, MA, USA, from 2001 to 2012. Public de-identified databases were used for this study, so informed consent and institutional review board (IRB) approval was waived. We were granted access to the database after completing a web-based training course based on the Cooperative Institutional Training Initiative (CITI program) entitled 'Data or Specimens Only Research' (Record ID: 36171671). This access was approved by the Institutional Review Boards of Beth Israel Deaconess Medical Center and MIT affiliates.

\section{Consent for publication}

Since not all patients were able to give their informed consent, the Ethics Committee waived this requirement. Informed consent was sought from all surviving patients as soon as they regained their mental competence.

\section{Availability of supporting data}

Data are available from the authors upon reasonable request.

\section{Competing interests}

The authors declare that there are no conflicts of interest regarding the publication of this article.

\section{Funding}

The authors received no specific funding for this work.

\section{Authors' contributions}

Name: Zehao Wu, MD.

Contribution: This author helped design the study, collect the data, perform the data analysis, tabulation, mapping, and write the manuscript.

Name: Huili Li, MD, PhD.

Contribution: This author helped design the study, analyze the statistical data, give statistical support, and write the manuscript.

Name: Kaihua Liao, MD.

Contribution: This author helped analyze and interpret the data, mapping, and critically review the manuscript.

Name: Yun Wang, MD, PhD.

Contribution: This author helped with the conception and design of the study, analysis and interpretation of the data, revising of the article, and final approval of the version to be published. 
Acknowledgements

No external funding or competing interests declared.

\section{References}

1. Inouye SK: Clarifying Confusion: The Confusion Assessment Method. ANN INTERN MED 1990, 113(12):941.

2. O'Regan NA, Fitzgerald J, Timmons S, O'Connell H, Meagher D: Delirium: A key challenge for perioperative care. INT J SURG 2013, 11(2):136-144.

3. Bilotta F, Lauretta MP, Borozdina A, Mizikov VM, Rosa G: Postoperative delirium: risk factors, diagnosis and perioperative care. MINERVA ANESTESIOL 2013, 79(9):1066.

4. Smulter N, Lingehall HC, Gustafson Y, Olofsson B, Engstrom KG: Delirium after cardiac surgery: incidence and risk factors. Interact Cardiovasc Thorac Surg 2013, 17(5):790-796.

5. Saczynski JS, Marcantonio ER, Quach L, Fong TG, Gross A, Inouye SK, Jones RN: Cognitive Trajectories after Postoperative Delirium. NEW ENGL J MED 2012, 367(1):30-39.

6. Bettelli G, Neuner B: Postoperative delirium: A preventable complication in the elderly surgical patient. Monaldi archives for chest disease 2017, 87(2):842.

7. Gosselt AN, Slooter AJ, Boere PR, Zaal IJ: Risk factors for delirium after on-pump cardiac surgery: a systematic review. CRIT CARE 2015, 19:346.

8. Santos E, Cardoso D, Neves H, Cunha M, Rodrigues M, Apóstolo J: Effectiveness of haloperidol prophylaxis in critically ill patients with a high risk of delirium. JBI Database of Systematic Reviews and Implementation Reports 2017, 15(5):1440-1472.

9. Sakusic A, O'Horo JC, Dziadzko M, Volha D, Ali R, Singh TD, Kashyap R, Farrell AM, Fryer JD, Petersen R et al: Potentially Modifiable Risk Factors for Long-Term Cognitive Impairment After Critical Illness: A Systematic Review. MAYO CLIN PROC 2018, 93(1):68-82.

10. Lee A, Mu JL, Joynt GM, Chiu CH, Lai VKW, Gin T, Underwood MJ: Risk prediction models for delirium in the intensive care unit after cardiac surgery: a systematic review and independent external validation. BRIT J ANAESTH 2017, 118(3):391-399.

11. Girard TD, Jackson JC, Pandharipande PP, Pun BT, Thompson JL, Shintani AK, Gordon SM, Canonico AE, Dittus RS, Bernard GR et al: Delirium as a predictor of long-term cognitive impairment in survivors of critical illness. CRIT CARE MED 2010, 38(7):1513-1520.

12. Leslie DL, Inouye SK: The importance of delirium: economic and societal costs. J AM GERIATR SOC 2011, 59 Suppl 2:S241-S243.

13. Dillon ST, Vasunilashorn SM, Ngo L, Otu HH, Inouye SK, Jones RN, Alsop DC, Kuchel GA, Metzger ED, Arnold SE et al: Higher CReactive Protein Levels Predict Postoperative Delirium in Older Patients Undergoing Major Elective Surgery: A Longitudinal Nested Case-Control Study. BIOL PSYCHIAT 2017, 81(2):145-153.

14. Li L, Wang C, Fang M, Xu H, Lu H, Zhang H: Effects of dexamethasone on post-operative cognitive dysfunction and delirium in adults following general anaesthesia: a meta-analysis of randomised controlled trials. BMC ANESTHESIOL 2019, 19(1):113.

15. Smith C, Erasmus PJ, Myburgh KH: Endocrine and Immune Effects of Dexamethasone in Unilateral Total Knee Replacement. The Journal of International Medical Research 2006, 34(6):603-611.

16. Holte K, Kehlet H: Perioperative single-dose glucocorticoid administration: pathophysiologic effects and clinical implications. J Am Coll Surg 2002, 195(5):694-712.

17. Dabul L, Droney A, Oms J, Sanchez-Gonzalez MA: Acute Changes in Mentation in a Patient with Hepatic Cirrhosis Treated with High Doses of Dexamethasone. Cureus 2017, 9(9):e1675.

18. Kenna HA, Poon AW, de Los Angeles CP, Koran LM: Psychiatric complications of treatment with corticosteroids: Review with case report. PSYCHIAT CLIN NEUROS 2011, 65(6):549-560.

19. Mardani D, Bigdelian H: Predictors and clinical outcomes of postoperative delirium after administration of dexamethasone in patients undergoing coronary artery bypass surgery. Int J Prev Med 2012, 3(6):420-427.

20. Sauer AM, Slooter AJ, Veldhuijzen DS, van Eijk MM, Devlin JW, van Dijk D: Intraoperative dexamethasone and delirium after cardiac surgery: a randomized clinical trial. ANESTH ANALG 2014, 119(5):1046-1052.

21. Mardani D, Bigdelian H: Prophylaxis of dexamethasone protects patients from further post-operative delirium after cardiac surgery: A randomized trial. J RES MED SCI 2013, 18(2):137-143.

22. Johnson AEW, Pollard TJ, Shen L, Lehman LH, Feng M, Ghassemi M, Moody B, Szolovits P, Anthony Celi L, Mark RG: MIMIC-III, a freely accessible critical care database. SCI DATA 2016, 3(1):160035. 
23. Zhang Z: Accessing critical care big data: a step by step approach. J THORAC DIS 2015, 7(3):238-242.

24. Vincent JL, Moreno R, Takala J, Willatts S, De Mendonça A, Bruining H, Reinhart CK, Suter PM, Thijs LG: The SOFA (Sepsis-related Organ Failure Assessment) score to describe organ dysfunction/failure. On behalf of the Working Group on Sepsis-Related Problems of the European Society of Intensive Care Medicine. INTENS CARE MED 1996, 22(7):707-710.

25. Metnitz PG, Moreno RP, Almeida E, Jordan B, Bauer P, Campos RA, lapichino G, Edbrooke D, Capuzzo M, Le Gall JR: SAPS 3-From evaluation of the patient to evaluation of the intensive care unit. Part 1: Objectives, methods and cohort description. Intensive Care Med 2005, 31(10):1336-1344.

26. Reade MC, O'Sullivan K, Bates S, Goldsmith D, Ainslie WRST, Bellomo R: Dexmedetomidine vs. haloperidol in delirious, agitated, intubated patients: a randomised open-label trial. Critical care (London, England) 2009, 13(3):R75.

27. Bledowski J, Trutia A: A Review of Pharmacologic Management and Prevention Strategies for Delirium in the Intensive Care Unit. PSYCHOSOMATICS 2012, 53(3):203-211.

28. Postoperative delirium in older adults: best practice statement from the American Geriatrics Society. J Am Coll Surg 2015, 220(2):136-148.

29. Ebersoldt M, Sharshar T, Annane D: Sepsis-associated delirium. Intensive Care Med 2007, 33(6):941-950.

30. Prasongsukarn K, Abel JG, Jamieson WR, Cheung A, Russell JA, Walley KR, Lichtenstein SV: The effects of steroids on the occurrence of postoperative atrial fibrillation after coronary artery bypass grafting surgery: a prospective randomized trial. $J$ Thorac Cardiovasc Surg 2005, 130(1):93-98.

31. Siddiqi N, Harrison JK, Clegg A, Teale EA, Young J, Taylor J, Simpkins SA: Interventions for preventing delirium in hospitalised nonICU patients. Cochrane Database Syst Rev 2016, 3:D5563.

32. Moheimani H, Yaseri M: Dexamethasone Reduces Postoperative Nausea in Pediatric Upper Endoscopy With Deep Sedation. $J$ PEDIATR GASTR NUTR 2019, 69(3):281-286.

33. Steiner LA: Postoperative delirium. Part 1. EUR J ANAESTH2011, 28(9):628-636.

34. Tremblay P, Gold S: Prevention of Post-operative Delirium in the Elderly Using Pharmacological Agents. Canadian geriatrics journal CGJ 2016, 19(3):113.

35. Liu Y, Li XJ, Liang Y, Kang Y: Pharmacological Prevention of Postoperative Delirium: A Systematic Review and Meta-Analysis of Randomized Controlled Trials. Evid Based Complement Alternat Med 2019, 2019:9607129.

36. Mariscalco GMP, Cottini MM, Zanobini MMP, Salis SM, Dominici CM, Banach MMP, Onorati FMP, Piffaretti GMP, Covaia GM, Realini MM et al: Preoperative Statin Therapy Is Not Associated With a Decrease in the Incidence of Delirium After Cardiac Operations. ANN THORAC SURG 2012, 93(5):1439-1447.

37. Moots RJ, Al-Saffar Z, Hutchinson D, Golding SP, Young SP, Bacon PA, McLaughlin PJ: Old drug, new tricks: haloperidol inhibits secretion of proinflammatory cytokines. ANN RHEUM DIS 1999, 58(9):585-587.

38. Kalisvaart KJ, de Jonghe JF, Bogaards MJ, Vreeswijk R, Egberts TC, Burger BJ, Eikelenboom P, van Gool WA: Haloperidol prophylaxis for elderly hip-surgery patients at risk for delirium: a randomized placebo-controlled study. J AM GERIATR SOC 2005, 53(10):16581666.

39. Nishimura K, Harigai M, Omori M, Sato E, Hara M: Blood-brain barrier damage as a risk factor for corticosteroid-induced psychiatric disorders in systemic lupus erythematosus. PSYCHONEUROENDOCRINO 2007, 33(3):395-403.

40. Wada K, Yamada N, Sato T, Suzuki H, Miki M, Lee Y, Akiyama K, Kuroda S: Corticosteroid-Induced Psychotic and Mood Disorders: Diagnosis Defined by DSM-IV and Clinical Pictures. PSYCHOSOMATICS 2001, 42(6):461-466.

41. Mclntosh T: Beta-endorphin, cortisol and postoperative delirium: a preliminary report. PSYCHONEUROENDOCRINO 1985, 10(3):303313.

42. Olsson T: Activity in the Hypothalamic-Pituitary-Adrenal Axis and Delirium. DEMENT GERIATR COGN1999, 10(5):345-349.

43. Mandell AJ, Chapman LF, Rand RW, Walter RD: Plasma Corticosteroids: Changes in Concentration after Stimulation of Hippocampus and Amygdala. SCIENCE 1963, 139(3560):1212.

44. JACOBSON L, SAPOLSKY R: The Role of the Hippocampus in Feedback Regulation of the Hypothalamic-Pituitary-Adrenocortical Axis*. ENDOCR REV1991, 12(2):118-134.

45. De Kloet ER, Vreugdenhil E, Oitzl MS, Joels M: Brain corticosteroid receptor balance in health and disease. ENDOCR REV1998, 19(3):269-301. 
46. Holmes MC, Yau JLW, French KL, SeckI JR: The effect of adrenalectomy on 5-hydroxytryptamine and corticosteroid receptor subtype messenger RNA expression in rat hippocampus. NEUROSCIENCE 1995, 64(2):327-337.

47. Holmes MC, French KL, Seckl JR: Dysregulation of diurnal rhythms of serotonin 5-HT2C and corticosteroid receptor gene expression in the hippocampus with food restriction and glucocorticoids. J NEUROSCI 1997, 17(11):4056-4065.

48. Schutsky K, Ouyang M, Castelino CB, Zhang L, Thomas SA: Stress and Glucocorticoids Impair Memory Retrieval via 2-Adrenergic, Gi/o-Coupled Suppression of cAMP Signaling. J NEUROSCI 2011, 31(40):14172-14181.

49. Newcomer JW, Craft S, Hershey T, Askins K, Bardgett ME: Glucocorticoid-induced impairment in declarative memory performance in adult humans. The Journal of neuroscience 1994, 14(4):2047-2053.

50. Lupien SJ, de Leon M, de Santi S, Convit A, Tarshish C, Nair NPV, Thakur M, McEwen BS, Hauger RL, Meaney MJ: Cortisol levels during human aging predict hippocampal atrophy and memory deficits. NAT NEUROSC/1998, 1(1):69-73.

51. Gurevich D, Siegel B, Dumlao M, Perl E, Chaitin P, Bagne C, Oxenkrug G: HPA axis responsivity to dexamethasone and cognitive impairment in dementia. PROG NEURO-PSYCHOPH 1990, 14(3):297.

52. Belanoff JK, Gross K, Yager A, Schatzberg AF: Corticosteroids and cognition. J PSYCHIATR RES 2001, 35(3):127-145.

53. Chau SY, Mok CC: Factors predictive of corticosteroid psychosis in patients with systemic lupus erythematosus. NEUROLOGY 2003 , 61(1):104-107.

54. Genis B, Cosar B: [Mania Associated with the Use of Bortezomib and Dexamethasone]. Turk Psikiyatri Derg 2018, 29(3):209-215.

55. Acute adverse reactions to prednisone in relation to dosage. Clinical pharmacology and therapeutics 1972, 13(5):694.

56. Chan L, French ME, Oliver DO, Morris PJ: High- and low-dose prednisolone. TRANSPL P1981, 13(1 Pt 1):336.

57. Galen DM, Beck M, Buchbinder D: Steroid psychosis after orthognathic surgery: a case report. J Oral Maxillofac Surg 1997, 55(3):294-297.

58. Prado CE, Crowe SF: Corticosteroids and Cognition: A Meta-Analysis. NEUROPSYCHOL REV2019, 29(3):288-312.

59. Kuhn E, Du X, McGrath K, Coveney S, O'Regan N, Richardson S, Teodorczuk A, Allan L, Wilson D, Inouye SK et al: Validation of a consensus method for identifying delirium from hospital records. PLOS ONE 2014, 9(11):e111823.

60. Chang JT, Atayee RS, Edmonds KP: Identifying Patterns of Delirium in Hospitalized Patients on Dexamethasone Using a Chart Abstraction Tool. Journal of Pain \& Palliative Care Pharmacotherapy 2018, 32(1):30-36.

61. Mu JL, Lee A, Joynt GM: Pharmacologic Agents for the Prevention and Treatment of Delirium in Patients Undergoing Cardiac Surgery: Systematic Review and Metaanalysis. CRIT CARE MED 2015, 43(1):194-204.

62. Dieleman JM: Intraoperative High-Dose Dexamethasone for Cardiac Surgery. JAMA 2012, 308(17):1761.

63. Shih A, Jackson KN: Role of corticosteroids in palliative care. J Pain Palliat Care Pharmacother 2007, 21(4):69-76.

64. Sandhu G, Farias AA, Ranade A, Meisels I: Altered mental status in a case of multiple myeloma not related to a metabolic cause. NDT Plus 2009, 2(5):434-435.

65. HOPKINS RO, WEAVER LK, POPE D, ORME JFJ, BIGLER ED, LARSON-LOHR V: Neuropsychological Sequelae and Impaired Health Status in Survivors of Severe Acute Respiratory Distress Syndrome. AM J RESP CRIT CARE 1999, 160(1):50-56.

66. Tao R, Wang X, Pang L, Cheng J, Wang Y, Gao G, Liu Y, Wang C: Pharmacologic prevention of postoperative delirium after on-pump cardiac surgery. MEDICINE 2018, 97(43):e12771.

\section{Tables}

Table 1. Baseline characteristics of the dexamethasone and non-dexamethasone groups before and after propensity score matching 


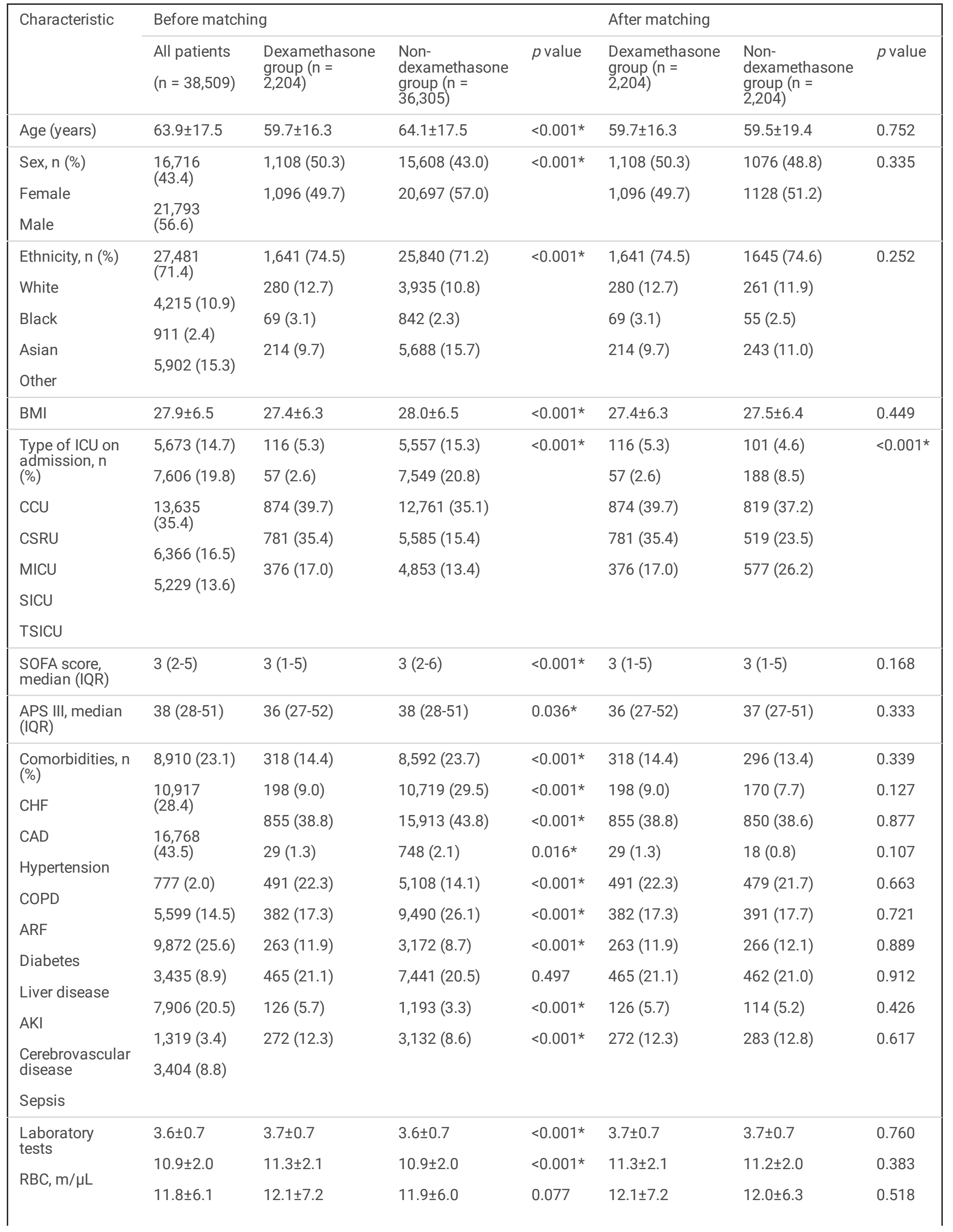




\begin{tabular}{|c|c|c|c|c|c|c|c|}
\hline Hemoglobin, & $215.6 \pm 103.0$ & $226.9 \pm 114.9$ & $214.9 \pm 102.2$ & $<0.001 *$ & $226.9 \pm 114.9$ & $226.8 \pm 108.8$ & 0.986 \\
\hline XRA K/W & $138.3 \pm 4.6$ & $138.0 \pm 5.1$ & $138.3 \pm 4.5$ & $0.006^{*}$ & $138.0 \pm 5.1$ & $138.1 \pm 4.8$ & 0.767 \\
\hline VDL, K/ML & $4.2 \pm 0.7$ & $4.1 \pm 0.6$ & $4.2 \pm 0.7$ & $<0.001^{*}$ & $4.1 \pm 0.6$ & $4.1 \pm 0.7$ & 0.748 \\
\hline Platelet, $\mathrm{K} / \mu \mathrm{L}$ & $8.3 \pm 0.9$ & $8.4 \pm 1.0$ & $8.3 \pm 0.9$ & $<0.001^{\star}$ & $8.4 \pm 1.0$ & $8.4 \pm 1.0$ & 0.260 \\
\hline DOC. & $23.6 \pm 4.4$ & $23.5 \pm 4.3$ & $23.6 \pm 4.4$ & 0.139 & $23.5 \pm 4.3$ & $23.5 \pm 4.5$ & 0.981 \\
\hline $\begin{array}{l}\mathrm{mEtass} / \mathrm{L} \text {, } \\
\mathrm{mEq} / \mathrm{m}\end{array}$ & $1.3 \pm 1.2$ & $1.1 \pm 1.1$ & $1.3 \pm 1.2$ & $<0.001^{*}$ & $1.1 \pm 1.1$ & $1.1 \pm 1.1$ & 0.755 \\
\hline Calcium, mg/dL & $23.5 \pm 19.2$ & $22.4 \pm 18.4$ & $23.6 \pm 19.2$ & $0.003^{*}$ & $22.4 \pm 18.4$ & $22.5 \pm 20.0$ & 0.827 \\
\hline Bicarbonate, & $144.9 \pm 67.1$ & $149.9 \pm 64.6$ & $144.6 \pm 67.3$ & $<0.001 *$ & $149.9 \pm 64.6$ & $150.2 \pm 80.7$ & 0.867 \\
\hline $\begin{array}{l}\text { mEq/L } \\
\text { Creatinine, } \\
\text { mg/dL }\end{array}$ & $2.1 \pm 1.5$ & $2.1 \pm 1.4$ & $2.1 \pm 1.5$ & 0.657 & $2.1 \pm 1.4$ & $2.1 \pm 1.4$ & 0.766 \\
\hline $\mathrm{BUN}, \mathrm{mg} / \mathrm{dL}$ & & & & & & & \\
\hline Glucose, mg/dL & & & & & & & \\
\hline $\begin{array}{l}\text { Lactate, } \\
\mathrm{mmol} / \mathrm{L}\end{array}$ & & & & & & & \\
\hline
\end{tabular}

Notes: Data are presented as mean \pm standard deviation, median (IQR), or $\mathrm{n}(\%)$. Comparisons were performed using the t-test, Kruskal Wallis or $\chi^{2}$ (or Fisher's exact) test. * Statistically significant $(P<0.05)$.

Abbreviations: $\mathrm{BMI}$, body mass index; ICU, intensive care unit; $\mathrm{CCU}$, coronary care unit; CSRU, cardiac surgery recovery unit; MICU, medical intensive care unit; SICU, surgical intensive care unit; TSICU, trauma/surgical intensive care unit; IQR, interquartile range; SOFA, Sequential Organ Failure Assessment; APS III, Acute Physiology Score III; CHF, congestive heart failure; CAD, coronary artery disease; COPD, chronic obstructive pulmonary disease; ARF, acute respiratory failure; AKI, acute kidney injury; RBC, red blood cell; WBC, white blood cell; BUN, blood urea nitrogen.

Table 2. Crude comparison of dexamethasone use with outcome measures in critically ill patients

\begin{tabular}{|c|c|c|c|c|c|c|}
\hline \multirow[t]{2}{*}{ Variables } & \multicolumn{3}{|l|}{ Before matching } & \multicolumn{3}{|l|}{ After matching } \\
\hline & $\begin{array}{l}\text { Dexamethasone } \\
\text { group }(n=2,204)\end{array}$ & $\begin{array}{l}\text { Non-dexamethasone } \\
\text { group }(n=36,305)\end{array}$ & $p$ value & $\begin{array}{l}\text { Dexamethasone } \\
\text { group }(n=2,204)\end{array}$ & $\begin{array}{l}\text { Non-dexamethasone } \\
\text { group }(n=2,204)\end{array}$ & $p$ value \\
\hline $\begin{array}{l}\text { Primary } \\
\text { outcome }\end{array}$ & $110(5.0)$ & $1,224(3.4)$ & $<0.001^{*}$ & $110(5.0)$ & $77(3.5)$ & $0.014^{*}$ \\
\hline \multicolumn{7}{|l|}{ Delirium, n (\%) } \\
\hline \multirow{2}{*}{$\begin{array}{l}\text { Secondary } \\
\text { outcomes }\end{array}$} & $331(15.0)$ & $4,085(11.3)$ & $<0.001^{\star}$ & $331(15.0)$ & $300(13.6)$ & 0.182 \\
\hline & $10.7(6.1-19.5)$ & $6.8(4.0-11.5)$ & $<0.001^{*}$ & $10.7(6.1-19.5)$ & $7(3.9-12.8)$ & $<0.001^{*}$ \\
\hline mortality, n (\%) & $2.9(1.4-6.8)$ & $2.1(1.2-4.0)$ & $<0.001^{*}$ & $2.9(1.4-6.8)$ & $2.1(1.3-4.5)$ & $<0.001 *$ \\
\hline \multicolumn{7}{|l|}{$\begin{array}{l}\text { Hospital LOS, } \\
\text { median (IQR) }\end{array}$} \\
\hline $\begin{array}{l}\text { ICU LOS, } \\
\text { median (IQR) }\end{array}$ & & & & & & \\
\hline
\end{tabular}

Notes: Data are presented as $n(\%)$ or median (IQR). Comparisons were performed using $\chi^{2}$ (or Fisher's exact) or Kruskal Wallis test. * Statistically significant $(P<0.05)$.

Abbreviations: LOS, length of stay; IQR, interquartile range.

Table 3. Association between dexamethasone use and delirium and hospital mortality 


\begin{tabular}{|c|c|c|c|c|}
\hline Outcomes & Group & Odds Ratio (or Hazard Ratio) & $95 \% \mathrm{Cl}$ & $p$ value \\
\hline \multicolumn{5}{|l|}{ Delirium } \\
\hline \multirow[t]{2}{*}{ Crude } & Non-dexamethasone & Ref & $1.23-1.84$ & $<0.001^{*}$ \\
\hline & Dexamethasone & 1.51 & & \\
\hline \multirow[t]{2}{*}{ Model 1} & Non-dexamethasone & Ref & $1.24-1.86$ & $<0.001 *$ \\
\hline & Dexamethasone & 1.52 & & \\
\hline \multirow[t]{2}{*}{ Model 2} & Non-dexamethasone & Ref & $1.18-1.78$ & $<0.001^{*}$ \\
\hline & Dexamethasone & 1.45 & & \\
\hline \multirow[t]{2}{*}{ Model 3} & Non-dexamethasone & Ref & $1.08-1.95$ & $0.014^{\star}$ \\
\hline & Dexamethasone & 1.45 & & \\
\hline \multicolumn{5}{|c|}{ Hospital mortality } \\
\hline \multirow[t]{2}{*}{ Crude } & Non-dexamethasone & Ref & $1.29-1.62$ & $<0.001^{*}$ \\
\hline & Dexamethasone & 1.45 & & \\
\hline \multirow[t]{2}{*}{ Model 1} & Non-dexamethasone & Ref & $1.35-1.70$ & $<0.001 *$ \\
\hline & Dexamethasone & 1.52 & & \\
\hline \multirow[t]{2}{*}{ Model 2} & Non-dexamethasone & Ref & $1.07-1.34$ & $0.002^{\star}$ \\
\hline & Dexamethasone & 1.20 & & \\
\hline \multirow[t]{2}{*}{ Model 3} & Non-dexamethasone & Ref & $1.02-1.40$ & $0.032^{\star}$ \\
\hline & Dexamethasone & 1.19 & & \\
\hline
\end{tabular}

Notes: The association between dexamethasone use and delirium incidence and hospital mortality in critically ill patients was analyzed using logistic regression models and Cox regression models, respectively. Model 1 was adjusted by: age, sex, ethnicity, BMI, and type of ICU on admission; Model 2, adding CHF, CAD, hypertension, COPD, ARF, diabetes, liver disease, cerebrovascular disease, sepsis, RBC, WBC, hemoglobin, platelets, sodium, potassium, calcium, creatinine, BUN, blood glucose, lactate, SOFA score and APS III to Model 1; Model 3 , a propensity score as mentioned above. * Statistically significant $(P<0.05)$.

Abbreviations: $\mathrm{Cl}$, confidence interval; BMI, body mass index; ICU, intensive care unit; SOFA, Sequential Organ Failure Assessment; APS III, Acute Physiology Score III; CHF, congestive heart failure; CAD, coronary artery disease; COPD, chronic obstructive pulmonary disease; ARF, acute respiratory failure; RBC, red blood cell; WBC, white blood cell; BUN, blood urea nitrogen.

Table 4. Association between dexamethasone use and delirium and hospital mortality stratified by types of ICU 


\begin{tabular}{|c|c|c|c|c|c|c|}
\hline & Crude & & & Adjust by Propensity Sc & & \\
\hline & $\begin{array}{l}\text { Odds Ratio (or Hazard } \\
\text { Ratio) }\end{array}$ & $95 \% \mathrm{Cl}$ & $p$ value & $\begin{array}{l}\text { Odds Ratio (or Hazard } \\
\text { Ratio) }\end{array}$ & $95 \% \mathrm{Cl}$ & $p$ value \\
\hline Delirium & & & & & & \\
\hline $\mathrm{CCU}$ & Ref & $0.48-$ & 0.587 & Ref & $0.32-9.86$ & 0.516 \\
\hline Non- & 1.32 & & 0.561 & 1.77 & $0.37-$ & 0.383 \\
\hline dexamethasone & Ref & $\begin{array}{l}0.3- \\
6.29\end{array}$ & $<0.001^{\star}$ & Ref & 13.16 & 0.085 \\
\hline 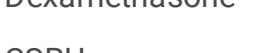 & 1.52 & $1.38-$ & 0.866 & 2.24 & $0.95-2.17$ & 0.624 \\
\hline CSRU & Ref & 2.41 & 0.900 & Ref & $0.63-2.18$ & 0.218 \\
\hline $\begin{array}{l}\text { Non- } \\
\text { dexamethasone }\end{array}$ & 1.83 & $\begin{array}{l}0.65- \\
1.44\end{array}$ & & 1.44 & $0.77-3.16$ & \\
\hline Dexamethasone & Ref & $0.61-$ & & Ref & & \\
\hline MICU & 0.97 & & & 1.17 & & \\
\hline Non- & Ref & & & Ref & & \\
\hline Dexamethasone & 1.03 & & & 1.56 & & \\
\hline SICU & & & & & & \\
\hline $\begin{array}{l}\text { Non- } \\
\text { dexamethasone }\end{array}$ & & & & & & \\
\hline Dexamethasone & & & & & & \\
\hline TSICU & & & & & & \\
\hline $\begin{array}{l}\text { Non- } \\
\text { dexamethasone }\end{array}$ & & & & & & \\
\hline Dexamethasone & & & & & & \\
\hline Hospital mortality & & & & & & \\
\hline $\mathrm{CCU}$ & Ref & $1.22-$ & $0.004^{\star}$ & Ref & $0.69-2.63$ & 0.386 \\
\hline Non- & 1.86 & & $<0.001^{*}$ & 1.35 & $1.06-6.84$ & $0.036^{*}$ \\
\hline 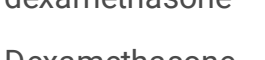 & Ref & 9.00 & $<0.001^{\star}$ & Ref & $1.42-2.22$ & $<0.001^{*}$ \\
\hline cद्us & 4.45 & $1.68-$ & $<0.001^{*}$ & 2.70 & $0.25-0.55$ & $<0.001^{\star}$ \\
\hline 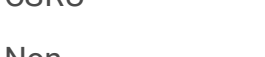 & Ref & & 0.973 & Ref & $0.63-1.41$ & 0.764 \\
\hline $\begin{array}{l}\text { Non- } \\
\text { dexamethasone }\end{array}$ & 1.93 & 0.52 & & 1.78 & & \\
\hline Dexamethasone & Ref & $0.72-$ & & Ref & & \\
\hline MICU & 0.38 & & & 0.38 & & \\
\hline Non- & Ref & & & Ref & & \\
\hline Dexamethasone & 1.01 & & & 0.94 & & \\
\hline SICU & & & & & & \\
\hline $\begin{array}{l}\text { Non- } \\
\text { dexamethasone }\end{array}$ & & & & & & \\
\hline Dexamethasone & & & & & & \\
\hline TSICU & & & & & & \\
\hline $\begin{array}{l}\text { Non- } \\
\text { dexamethasone }\end{array}$ & & & & & & \\
\hline
\end{tabular}


Notes: The association between dexamethasone use and delirium incidence and hospital mortality in critically ill patients was analyzed using logistic regression models and Cox regression models, respectively. Propensity score was calculated according to age, gender, ethnicity, BMI, SOFA score, APS III, CHF, CAD, hypertension, COPD, ARF, diabetes, liver disease, cerebrovascular disease, sepsis, RBC, WBC, hemoglobin, platelets, sodium, potassium, calcium, creatinine, BUN, blood glucose and lactate. * Statistically significant $(P<0.05)$.

Abbreviations: CI, confidence interval; ICU, intensive care unit; CCU, coronary care unit; CSRU, cardiac surgery recovery unit; MICU, medical intensive care unit; SICU, surgical intensive care unit; TSICU, trauma/surgical intensive care unit; BMI, body mass index; SOFA, Sequential Organ Failure Assessment; APS III, Acute Physiology Score III; CHF, congestive heart failure; CAD, coronary artery disease; COPD, chronic obstructive pulmonary disease; ARF, acute respiratory failure; RBC, red blood cell; WBC, white blood cell; BUN, blood urea nitrogen.

\section{Figures}

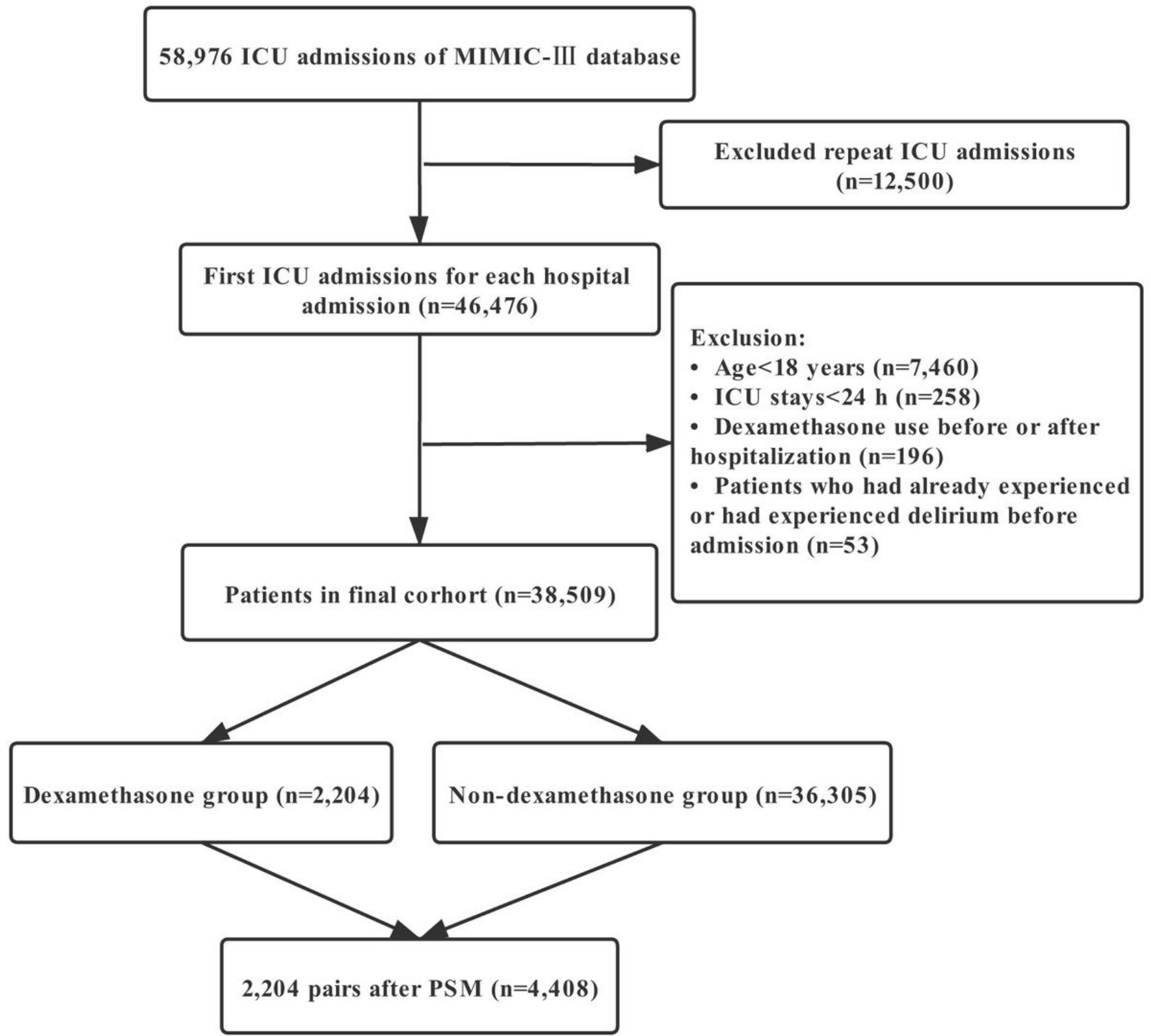

Figure 1

Flowchart of included subjects. MIMIC III: Medical Information Mart for Intensive Care III; ICU: intensive care unit; PSM: propensity score matching 


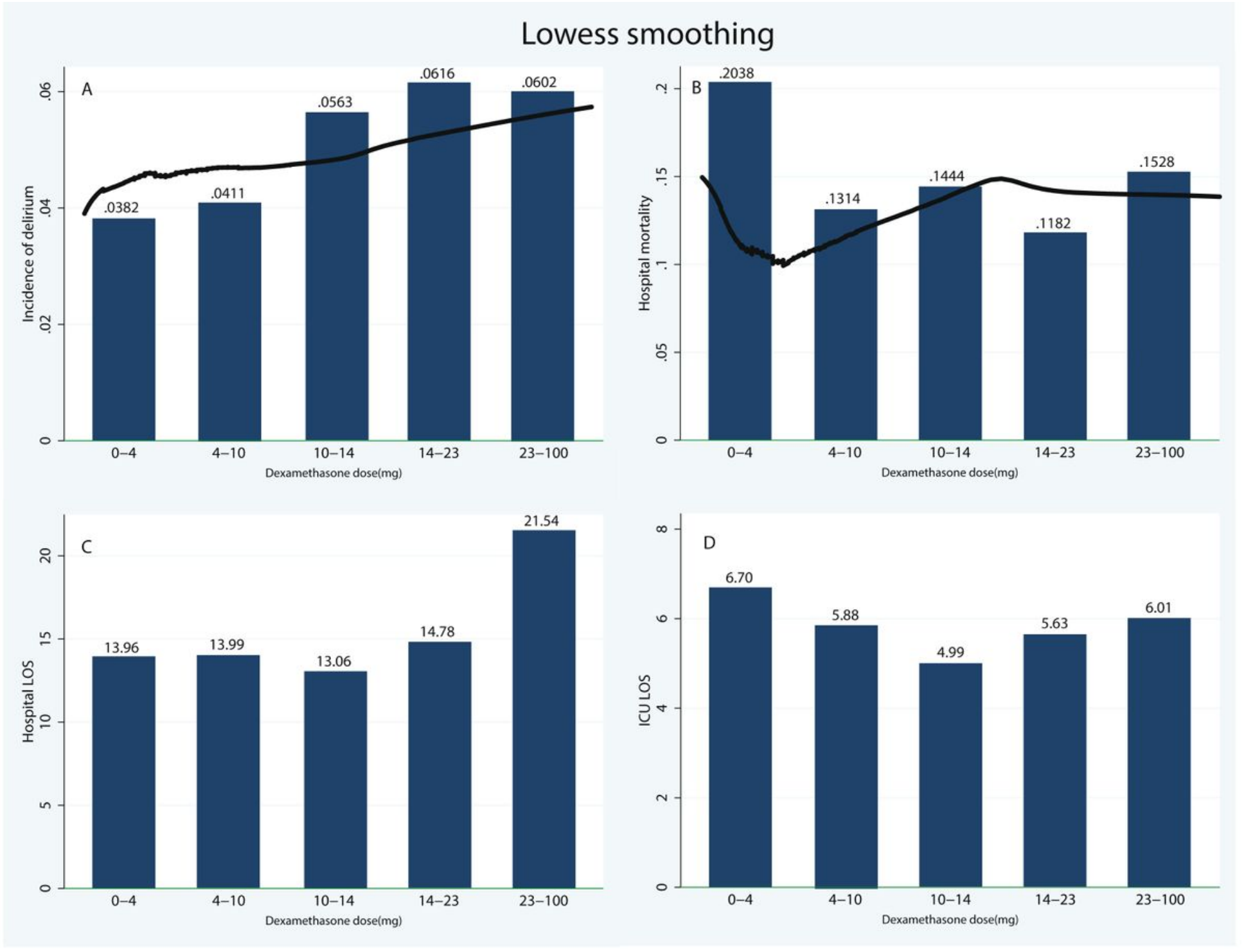

Figure 2

Effect of different doses of dexamethasone on primary and secondary outcomes: (A, B) differences in the incidence of delirium and inhospital death among patients using different doses were confirmed by fitting with a Lowess smoothing and logistic regression technique; (C, D) the optimal value of dexamethasone dosage to minimize the hospital and ICU LOS was confirmed by histogram. ICU, intensive care unit; LOS, length of stay. 